Acta Crystallographica Section D

Biological

Crystallography

ISSN 1399-0047

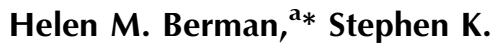
Burley, ${ }^{a}$ Gerard J. Kleywegt, ${ }^{b}$ Haruki Nakamurac ${ }^{c}$ and John L. Markley

a Department of Chemistry and Chemical Biology, Center for Integrative Proteomics Research, Rutgers, The State University of New Jersey, 174 Frelinghuysen Road, Piscataway, NJ 08854, USA, bPDBe, European Molecular Biology Laboratory-European Bioinformatics Institute, Cambridge CB10 1SD, UK, ${ }^{\mathrm{C}} \mathrm{PDBj}$, Institute for Protein Research, Osaka University, 3-2 Yamadaoka, Suita, Osaka, 565-0871, Japan, and ${ }^{\mathbf{d}}$ BioMagResBank, Department of Biochemistry, University of Wisconsin-Madison, Madison, WI 53706, USA

Correspondence e-mail:

berman@rcsb.rutgers.edu

Received 7 June 2014

Accepted 12 September 2014

\section{Response to On prompt update of literature references in the Protein Data Bank}

The wwPDB responds to the article On the prompt update of literature references in the Protein Data Bank [Wlodawer (2014), Acta Cryst. D70, 2779].

The wwPDB receives publication dates and citation information from authors, several journals, the user community, and additionally scans the literature for publications. The journals that currently provide related citation information either in advance or at the time of publication include IUCr Journals (Acta Crystallographica Sections $A-F$, IUCrJ, Journal of Applied Crystallography, Journal of Synchrotron Radiation); Journal of Biological Chemistry; Journal of Molecular Biology; Proceedings of the National Academy of Sciences; Nature journals; Science; Proteins: Structure, Function, and Bioinformatics; and Protein Science.

For each weekly update of the PDB archive, all files scheduled for release or modification are checked and validated one final time. Authors may be contacted to resolve any issues that may arise while preparing the entries for release. If this is not accomplished in time for that release cycle, the entry may be scheduled for release at a later date.

The preparation and packaging of the weekly PDB update begins Thursday afternoon (local time at each wwPDB member site), and the files are publicly released on the following Wednesday at 00:00 UTC (Coordinated Universal Time).

The wwPDB encourages journals to provide citation information [paper title, author list, related PDB ID(s), DOI, and publication date] to deposit@wwpdb.org, ideally at least two weeks in advance of publication of a structure report so that the release of the corresponding PDB entry can be timed accordingly.

\section{References}

Wlodawer, A. (2014). Acta Cryst. D70, 2779. 